\title{
Meduloblastoma: aspectos histológicos, moleculares e imunopatológicos
}

\section{Medulloblastoma: histological, molecular and immunopathological aspects}

\author{
Victor Hugo Clebis ${ }^{1}$; Karina Maturana Pinheiro ${ }^{1}$; Andressa Busetti Martins ${ }^{1}$; \\ Danilo Koiti Matsuda Feltran ${ }^{1}$; Raquel Mitie Harano ${ }^{1}$; Patricia Midori Murobushi \\ Ozawa $^{2}$; Carolina Batista Ariza ${ }^{3}$; Gabriela Gonçalves de Oliveira ${ }^{4}$; Maria Angelica \\ Ehara Watanabe ${ }^{5}$
}

\begin{abstract}
Resumo
O meduloblastoma é um tumor cerebelar caracterizado como tumor neuroectodérmico primitivo prevalente em crianças, sendo as do sexo masculinos as mais afetadas. Com relação à classificação histológica, existem cinco variações: clássico, desmoplásico, anaplásico, células gigantes e de extensa nodularidade. Muitos estudos relatam que a patogênese do meduloblastoma está relacionada com mutações em fatores de crescimento do SNC, sendo que as principais vias envolvidas são: Sonic Hedgehog, NOTCH, WNT e OTX. Ainda, com respeito à imunologia, pacientes com meduloblastoma apresentaram alta taxa de IFN- $\gamma$ no soro e células $T_{H} 17$ no sangue periférico, e foi observado que o TGF- $\beta$ tem sido associado à estimulação mitogênica, o que pode estar relacionado à patogênese da doença. A predominância de uma resposta $T_{H} 1$ relacionada à sobrevivência também foi relatada. O desenvolvimento terapêutico para o meduloblastoma, apesar de limitado, é significativo, uma vez que este vem apresentando melhora na sobrevida de seus pacientes. Entretanto, um maior conhecimento dos mecanismos envolvidos na imunopatogênese é necessário para o desenvolvimento de novos fármacos e formas de tratamento.
\end{abstract}

Palavras-chave: Tumor cerebelar. Vias de sinalização. Imunopatologia.

\begin{abstract}
Medulloblastoma is a cerebellar tumor classified as primitive neuroectodermal tumor and is prevalent in children, especially male. With regard to histological classification, there are five variations: classical, desmoplastic, anaplastic, large-cell variant and with extensive nodularity. Several studies have reported that medulloblastoma pathogenesis is related to mutations in CNS growth factors, and the main pathways involved are Sonic Hedgehog, NOTCH, WNT, and OTX. Also regarding the immunology, patients with medulloblastoma have a high serum concentration of INF- $\gamma$ and $\mathrm{T}_{\mathrm{H}} 17$ cells in peripheral blood, and it
\end{abstract}

${ }^{1}$ Graduando do curso de Biomedicina. Estagiário do Laboratório de Bacteriologia Básica e Aplicada do Departamento de Microbiologia da Universidade Estadual de Londrina.

${ }^{2}$ Mestranda do programa de Pós-Graduação em Patologia Experimental da Universidade Estadual de Londrina.

${ }^{3}$ Biomédica. Doutora em Biologia Molecular pela Universidade Federal de São Paulo, Brasil. Pós Doc do Laboratório Estudos e Aplicações de Polimorfismos de DNA. Departamento de Ciências Patológicas. Universidade estadual de Londrina.

${ }^{4}$ Doutora em Patologia Experimental pela Universidade Estadual de Londrina. Professora do Departamento de Ciências Patológicas.

${ }^{5}$ Doutora em Ciências Bioquímica pela Universidade de São Paulo, Brasil. Professor Associado do Departamento de Ciências Patológicas da Universidade Estadual de Londrina. E-mail: maewatuel@gmail.com 
was observed that TGF- $\beta$ has been associated with mitogenic stimulation, and possibly associated to the pathogenesis of this disease. The prevalence of a $\mathrm{T}_{\mathrm{H}} 1$ response related to the survival was also described. The development of therapies for medulloblastoma treatment, though limited, is significant, as they result in an improvement in the patient's survival. However, a better understanding of the mechanism involved in its immunopathogenesis is still necessary for the development of new drugs and ways of treatment.

Keywods: Primary health care. Child health. Continuity of patient care.

\section{Introdução}

O meduloblastoma (MB) é classificado pela Organização Mundial da Saúde (OMS) como uma neoplasia maligna cerebelar independente (PIZZO; POPLACK, 1997). Conforme a Associação Americana de Tumores Cerebrais (ABTA), o MB é um tumor cerebelar que pode desenvolver-se abaixo do tentorium cerebelli, uma extensão da dura-máter que separa o cérebro do cerebelo e geralmente surge no vérmis cerebelar delimitando os lóbulos do cerebelo (BOUFFET et al., 1991; FRIEDMAN et al., 1991). É considerada a neoplasia maligna primária mais comum do sistema nervoso central em crianças, e acomete principalmente a faixa etária de cinco a doze anos, apresentando uma maior incidência no sexo masculino (PAULINO, 2002).

A presença do tumor causa hidrocefalia pelo bloqueio do fluxo do líquido cefalorraquidiano causado pela massa do tumor (CRAWFORD; MACDONALD; PACKER, 2007), e é relacionada a episódios de cefaleia, vômito, ataxia e náusea (HALPERIN; FRIEDMAN, 1996). Os sintomas podem também ser relacionados com a disseminação de células cancerígenas pelo líquido cefalorraquidiano, como a paralisia de nervos cranianos devido à disseminação leptomeningea (MUOIO, 2010).

O desenvolvimento de novas terapias e técnicas possibilitou uma melhoria tanto na efetividade quanto na qualidade de vida dos pacientes (HELSETH et al., 1999; WALTER et al., 1999), no entanto, aproximadamente um terço dos pacientes ainda permanece incurável, e os tratamentos atuais têm deixado sequelas significativas nos sobreviventes (GILBERTSON; ELLISON, 2008).
Apesar de macroscópica e radiologicamente os meduloblastomas (MBs) apresentarem-se sempre como uma massa, histológica e molecularmente os MBs podem se apresentar de diversas maneiras. Segundo a OMS, há cinco variantes em relação à análise histológica (LOUIS et al., 2007). Além da classificação histológica, os casos de MBs também podem ser caracterizados conforme o perfil de expressão gênica em subgrupos moleculares, onde há desregulação de vias de sinalização específicas como a Sonic Hedgehog (SHH), Wingless (WNT) (NORTHCOTT et al., 2011; THOMPSON et al., 2006), NOTCH (XU et al., 2009) e OTX (ADAMSON et al., 2010).

Devido ao fato de os MBs possivelmente terem origem a partir de mutações em vias de sinalização relacionadas ao desenvolvimento do cerebelo (CARLOTTI JUNIOR; SMITH; RUTKA, 2008; LI; LAU; NG, 2013), diversas estratégias foram desenvolvidas para o tratamento desta doença baseados na inibição dos efeitos de tais mutações (GARBER, 2008; MUOIO, 2010). No entanto, existem ainda muitos aspectos a serem esclarecidos.

Neste contexto, o objetivo do presente trabalho é evidenciar a classificações histológica e molecular do MB, bem como abordar os aspectos imunopatológicos desta doença.

\section{Classificação Histológica do Meduloblastoma}

Os aspectos clínicos, radiológicos e macroscópicos apresentam-se relativamente semelhantes no $\mathrm{MB}$, no entanto, os aspectos histológicos não apresentam a mesma similaridade (MIN et al., 2006; SZYMAS et al., 1987). Assim 
sendo, os MBs apresentam-se como tumores heterogêneos, com uma tendência à diferenciação celular divergente, sendo, predominantemente, neuronal e, menos frequentemente, a glial (KLEIHUES; BURGER; SCHEITHAUER, 1993; RORKE, 1983; SCHIFF; WEN, 1995). Tais subtipos apresentam divergências em relação à frequência, apresentação clínica e prognóstico (FEIDEN; FEIDEN, 2008).

A mais recente classificação da OMS dos tumores do sistema nervoso central (SNC), de 2007, lista o $\mathrm{MB}$, definindo cinco variantes histológicas: clássico, desmoplásico, extensa modularidade, células gigantes e anaplásico (LOUIS et al., 2007). As variantes mais comuns são a clássica e a desmoplásica, sendo que, histologicamente, todas elas correspondem ao grau IV de malignidade, podendo ter um comportamento biológico diverso (KLEIHUES; BURGER; SCHEITHAUER, 1993).

OMBclássico, o qualapresenta uma caracterização clínica de variante mais frequente, é constituído de células pequenas redondas ou elipsoides, com uma elevada razão núcleo/citoplasma (GILBERTSON; ELLISON, 2008). No subtipo desmoplásico há células em constante proliferação, sendo que essa variante abrange clinicamente $15 \%$ dos MBs. Ele está relacionado à idade adulta e a um bom prognóstico (KATSETOS et al., 1989), provavelmente por ser um tumor mais delimitado, além da sua tendência a uma localização superficial nos lobos laterais do cerebelo, favorecendo a ressecção completa (KLEIHUES; BURGER; SCHEITHAUER, 1993; PETERSON; WALKER, 1995).

O subtipo de extensiva nodularidade é caracterizado histologicamente como células ganglionares, contendo grandes e numerosos nódulos com diferenciação neurocítica (SURESH et al., 2004; GILBERTSON; ELLISON, 2008). É determinado clinicamente como raro, apresentandose mais frequentemente em crianças com idade anterior a três anos, e são consideradas de melhor prognóstico, quando comparado ao do tipo clássico.
Esse subtipo também pode ser considerado como uma variante do tipo desmoplásico (ELLISON, 2002; GIANGASPERO et al., 1999; KATSETOS et al., 1989; PEARL; TAKEI, 1981).

O subtipo de células gigantes tem como característica histológica a presença de células redondas com aumento do volume celular, um único nucléolo proeminente, citoplasma abundante, um alto índice mitótico com polimorfismo nuclear e alta frequência de corpos apoptóticos agrupados ou isolados, sendo esses índices mais elevados do que em outros subtipos de MBs (ELLISON, 2002; GIANGASPERO et al., 1992; MCMANAMY et al., 2003). O subtipo células gigantes representa apenas 4\% dos MBs (ELLISON, 2002).

O último subtipo é o anaplásico, cujas células revelam-se em formas esféricas, nucléolo proeminente e atipia celular marcante. Essa variante compõe aproximadamente $24 \%$ dos casos, e está relacionada à agressividade clínica (EBERHART et al., 2002), apresentando em crianças um pior prognóstico do que aqueles com tumores clássicos (MCMANAMY et al., 2003).

Devido a incerteza sobre a histogênese dos MBs, existem atualmente quatro teorias na tentativa de esclarecê-la. A primeira defende que sua origem ocorre durante o período fetal na camada granular externa do cerebelo, supondo, então, uma diferenciação neuronal desta neoplasia (KATSETOS; BURGER, 1994). Já a segunda é baseada no princípio do tumor primitivo neuroectodérmico (PNET), e cita que os MB são oriundos das células da matriz subependimal (VANDENBERG; HERMAN; RUBINSTEIN, 1987). O terceiro conceito propõe que sua gênese se dá a partir de mais de uma célula, levantando a hipótese de que MBs clássicos provêm da matriz ventricular, enquanto a camada granular externa origina a variante desmoplásica (YACHNIS; RORKE; TROJANOWSKI, 1994).

A última e mais recente teoria para a origem dos MBs foi desenvolvida devido à recente descoberta de células tronco pluripotentes induzidas (iPS). 
Esta teoria é baseada no conceito de que células totalmente diferenciadas e maduras adquirem, durante a transformação neoplásica, um fenótipo multipotente primitivo, podendo gerar então o MB (RODINI et al., 2010).

\section{Classificação Meduloblastoma}

Molecular

do

O MB, como outros tipos de câncer, provém de um desequilíbrio entre os promotores e supressores da divisão celular. No caso do $\mathrm{MB}$, tal desequilíbrio está associado às vias de sinalização, segundos mensageiros e fatores de transcrição presentes na maturação do cerebelo. Assim, estudos moleculares, como a análise genômica, permitem identificar moléculas e vias relacionadas com o surgimento desta neoplasia e tal conhecimento, por sua vez, possibilita o desenvolvimento de fármacos e formas de tratamento que podem aumentar a sobrevida (THOMPSON et al., 2006; LI; LAU; NG, 2013).

É conhecido que o MB possui diferentes vias de tumorigênese, decorrentes de mutações nas vias de formação do cerebelo (CARLOTTI; SMITH; RUTKA, 2008). Isso, juntamente com dados sobre as diferentes formas histológicas, resulta na consideração do $\mathrm{MB}$, como uma doença com subgrupos molecularmente distintos, que são diferenciados também quanto a fatores como dados demográficos, prognóstico, anormalidades genéticas, entre outros (LI; LAU; NG, 2013).

Algumas vias já estão associadas com os tipos de organização histológica desta neoplasia. As principais vias envolvidas com a patogênese do MB são a Hedgehog, Wingless (WNT) (THOMPSON et al., 2006; NORTHCOTT et al., 2011) e NOTCH (XU et al., 2009). Porém, há evidências sobre uma via dependente do gene OTX2 (orthodenticle homeobox 2) capaz de causar a doença independentemente de distúrbios nas demais vias (ADAMSON et al., 2010).

\section{Via Hedgehog}

Hedgehogs são glicoproteínas envolvidas no crescimento de vários tecidos, tais como pulmonar, gonadal e nervoso, que interagem com receptores específicos que vão resultar na regulação de fatores de transcrição da família GLI (Gliomaassociated oncogene homolog) (STECCA; RUIZ; ALTABA, 2002). No caso do desenvolvimento do cerebelo, o Sonic ( $\mathrm{SHH}$ ), é secretado pelas células de Purkinje e interage com receptores PATCHED (PTCH) (CARLOTTI; SMITH; RUTKA, 2008) das células granulares, inibindo sua função inibida e dessa forma, a SMOOTHENED (SMO) é ativada (CHEN; STRUHL, 1996). A proteína SMO é responsável pela transdução do sinal da $\mathrm{SHH}$, (STECCA; RUIZ; ALTABA, 2002), regulando a liberação de fatores de transcrição da família GLI (DI MARCOTULLIO et al., 2006; YOON et al., 2009), que resultam na transcrição e tradução de genes promotores da divisão celular (DELLOVADE et al., 2006).

A via SHH apresenta vários componentes, o que possibilitou o estudo de fármacos e tratamentos para combater o MB proveniente desta via. Entre tais estudos, podemos destacar o tratamento com a ciclopamina, um antagonista da proteína SMO (TAIPALE et al., 2000) assim como outros tratamentos, como a inibição do fator GLI1 (GARBER, 2008). Esta via está relacionada à variante desmoplásica (KOOL et al., 2012).

\section{Via Wingless (WNT)}

As proteínas Wingless regulam 4 vias de sinalização, mas a mais relacionada ao $\mathrm{MB}$ e a mais estudada também, é a via canônica. Esta via é ativada pela interação da glicoproteína WNT com seu receptor, formado pela junção da proteína Frizzled com proteínas da família LRP (low-density lipoprotein receptor-related protein), o que gera o acúmulo da proteína beta-catenina (também conhecida como CTNNB1) (JOHNSON; RAJAMANNAN, 2006). Uma vez livre, a Beta- 
catenina migra para o núcleo, onde interage com os fatores de transcrição como LEF (Lymphoid enhancer fator) (BEHRENS et al., 1996) e TCF (T-cell Factor) (NOVAK; DEDHAR, 1999), resultando finalmente na transcrição e tradução de proteínas como c-MYC (Avian myelocytomatosis virus oncogene cellular homolog) (HE et al., 1998) e a ciclina D1 (TETSU; MCCORMICK, 1999). Sem a ativação, o complexo da beta-catenina sofre degradação via proteassoma (JOHNSON; RAJAMANNAN, 2006).

Cerca de $25 \%$ dos pacientes com MB apresentam algum tipo de mutação nesta via, e estes pacientes geralmente possuem um bom prognóstico (CLIFFORD et al., 2006). Um exemplo de estudo sobre o tratamento de MB originados por mutações na via WNT tem relação com o gene c-MYC, e mostrou expressão aumentada deste gene em $72 \%$ dos casos de MB. (ZHANG et al., 2006).

\section{Via NOTCH}

Essa via foi relacionada com o desenvolvimento neural em 2000 e com o MB apenas em 2004 (YOKOTA et al., 2004), e, embora seja apontada como uma possível via para o tratamento de MBs (FAN et al., 2004), ainda há necessidade de melhor entendimento e estudos a respeito deste assunto.

A família de receptores NOTCH, quando ativados por seus ligantes, resulta em duas proteólises consecutivas, que irão culminar na liberação do domínio interno. Este por sua vez é translocado para o núcleo, onde se complexa com o fator de transcrição CLS (CBF1/Su(H)/LAG-1) (SJOLUND et al., 2005), levando a transcrição de genes relacionados ao ciclo celular, como o p21 (RANGARAJAN et al., 2001) e a ciclina D1(RONCHINI; CAPOBIANCO, 2001).

Fan e colaboradores mostraram que o receptor NOTCH 1 e o NOTCH 2 possuem atividades opostas, uma vez que o NOTCH 1, ao contrário do NOTCH 2, está relacionado com a inibição da proliferação celular e sua expressão não é detectada em casos de MB. E, além disso, eles observaram que a atividade do NOTCH 1 suprime a expressão de genes alvos da via SHH, enquanto o NOTCH 2 regula positivamente os mesmos genes.

\section{Genes OTX}

Outra via envolvida na patogênese do MB parece estar relacionada com mutações nos genes da família OTX, principalmente os genes OTX1 e OTX2 (DE HAAS et al., 2006). Sabe-se que a ativação dos genes OTX1 e OTX2 estão relacionadas com a diferenciação do sistema nervoso central (LARSEN et al., 2010). No entanto, ainda não está bem elucidado o papel destes genes na patogênese do MB, apesar de já se saber que a via dos genes OTX1 e OTX2 tem seu potencial oncogênico devido ao aumento da expressão do gene c-MYC (ADAMSON et al., 2010) e a interação com a via WNT através do fator Wnt5 (MORGAN et al., 1999).

Além disso, há indícios de que MBs OTX2 positivos estão localizados preferencialmente no vérmis cerebelar (DE HAAS et al., 2006). A literatura também mostra que o ácido transretinóico é capaz de silenciar o gene OTX2, induzindo a apoptose de células do MB, sendo indicado como uma possível terapia (DI et al., 2005; CHANG et al., 2007; HALILAGIC et al., 2007).

\section{Imunopatologia do Meduloblastoma}

A relação entre inflamação, imunidade e câncer é amplamente aceita, porém os mecanismos mediadores dessa relação ainda permanecem desconhecidos. Já é bem conhecido que o fator de crescimento transformante-beta (TGF- $\beta$ ) pode atuar como um oncogene durante a progressão tumoral. Em MB, o efeito do TGF- $\beta$ tem sido associado à estimulação mitogênica (JENNINGS et al., 1994). E, além disso, o TGF- $\beta$ por possuir propriedades anti-inflamatórias e imunossupressoras pode 
favorecer o crescimento do tumor inibindo a resposta imune contra as células tumorais (FOGARTY; KESSLER; WECHSLER-REYA, 2005). Esta citocina também é conhecida por atuar na transição epitelial para mesenquimal, podendo promover a metástase (THIERY, 2002). Neste contexto, Aref e colaboradores (2013) avaliaram o Smad3, um componente principal da via de sinalização de TGF- $\beta$, utilizando-se a técnica de imunohistoquímica em tecidos humanos malignos, e foi demonstrado que o mesmo se correlaciona com a metástase e sobrevivência em MB.

É conhecido que receptores de proteínas quinases acoplados à proteína G (GRKs) têm sido implicados na regulação da metástase. Entretanto, a função específica e a regulação de GRKs em MB ainda não foram esclarecidas. Yuan e colaboradores (2013) observaram a coexpressão de receptor de fator de crescimento derivado de plaquetas (PDGFRA), receptor 4 de quimiocina da família CXC (CXCR4) e quimiocina ligante do receptor CXCR4 (CXCL12) no subtipo MB SHH em comparação com o MB não-SHH (cinco, sete, e mais de cinco vezes, respectivamente). Os autores demonstram que um novo mecanismo de GF receptor/PDGFR-Src induz a desregulação da sinalização do CXCR4, o que promove a migração de células do MB.

Atualmente, o sucesso terapêutico nesse tipo de tumor é limitado, especialmente em crianças menores de idade e com radioterapia induzidas por longo tempo. A fim de evitar ou atrasar a radioterapia cranioespinhal, doses elevadas de quimioterapia, seguida de transplante autólogo de células estaminais (HSCT), tornou-se uma modalidade de tratamento. Há poucos dados sobre o microambiente imunológico hospedeiro em pacientes com MB, como também há poucos estudos sobre a expressão das citocinas e reconstituição imunológica em pacientes com MB submetidos a transplante. Wiegering e colaboradores avaliaram prospectivamente a função imunológica após o transplante autólogo em dezessete crianças com MB. Anteriormente ao transplante, os pacientes mostraram níveis mais elevados de interferon gama (IFN- $\gamma$ ) no soro, assim como um maior número de células $\mathrm{T}$ positivas para o IFN. Após o transplante, este efeito foi ainda mais pronunciado. Os pacientes com maior número de células T positivas para o IFN- $\gamma$ e TNF (Tumor necrosis fator) tiveram um resultado mais favorável em todos os momentos analisados. Foi encontrado também predominância da resposta $\mathrm{T}_{\mathrm{H}} 1$ relacionada à sobrevivência.

Foi realizado um estudo comparando dezessete voluntários saudáveis e vinte e três pacientes com MB, e observou-se uma proporção mais elevada de células $T_{H} 17$ no sangue periférico dos pacientes que apresentavam o tumor. Também estava presente o aumento de porcentagem de células $T_{H} 17$ nas células $T$ que infiltram o MB, em relação ao controle. Além disso, foi verificado aumento da concentração sérica de citocinas relacionadas ao perfil $\mathrm{T}_{\mathrm{h}} 17$ como a IL-17 e IL-23, bem como aumento dos níveis de RNAm destas citocinas em tecidos do tumor de pacientes com MB. Neste contexto, tem sido relatado que a IL17 altera a vigilância imunológica (NUMASAKI et al., 2005) e a IL23 promove a incidência e crescimento do tumor (LANGOWSKI; KASTELEIN; OFT, 2007). Dessa forma, os resultados indicam que as células $\mathrm{T}_{\mathrm{H}} 17$ podem contribuir para a patogênese do MB (ZHOU; SHA; ZHU, 2010).

\section{Conclusão}

O MB é um tumor cerebelar que afeta principalmente crianças, apresentando grande relevância clínica devido a sua alta taxa de mortalidade e mau prognóstico. Assim, muitos estudos acerca de todo o desenvolvimento tumoral estão sendo realizados, a fim de possibilitar uma melhora no prognóstico e quadro clínico dos pacientes. Entretanto, apesar de todos os avanços 
observados na sua abordagem terapêutica, os numerosos estudos ainda apresentam muitos dados controversos, limitando o conhecimento a respeito da doença e o desenvolvimento científico que isso possibilitará. Sendo assim, a imunopatologia da doença é uma questão ainda pouco esclarecida e desenvolvida, sendo que, o conhecimento do perfil do paciente ainda é muito escasso, sendo necessários mais investimentos nessa conduta.

Sendo o MB um tumor agressivo de alta capacidade de disseminação, é necessário que haja mais investigações a respeito dos componentes envolvidos, ressaltando os aspectos celulares e moleculares das vias de sinalização, a fim de proporcionar novos alvos terapêuticos.

\section{Referências}

ADAMSON, D. C.; SHI, Q.; WORTHAM, M.; NORTHCOTT, P. A.; DI, C.; DUNCAN, C. G.; LI, J.; MCLENDON, R. E.; BIGNER, D. D.; TAYLOR, M. D.; YAN, H. OTX2 is critical for the maintenance and progression of Shh-independent medulloblastomas. Cancer Research, Chicago, v. 70, n. 1, p. 181-191, 2010.

AREF, D.; MOFFATT,C. J.; AGNIHOTRI, S.; RAMASWAMY,V.; M. DUBUC,A.; NORTHCOTT,P. A.; TAYLOR,M. D.; PERRY,A.; OLSON, J. M.; EBERHART,C. G.; CROUL,S. E. Canonical TGF-beta pathway activity is a predictor of SHH-driven medulloblastoma survival and delineates putative precursors in cerebellar development. Brain Pathology, Zürich, v. 23, n. 2, p. 178-191, 2013.

BEHRENS, J.; VON KRIESJ, P.; KUHL, M.; BRUHN,L.; WEDLICH,D.; GROSSCHEDL,R.; BIRCHMEIER,W.Functional interaction of betacatenin with the transcription factor LEF-1. Nature, London, v. 382, n. 6592, p. 638-642, 1996.

BOUFFET, E.; FRAPPAZ, D.; CARRIE, C.; FREYCON, F.; BRUNAT-MENTIGNY, $M$. Medulloblastoma. Pediatrie, Bucharest, v. 46, n. 1, p. 71-78, 1991.
CARLOTTI JUNIOR, C. G.; SMITH, C.; RUTKA, J. T. The molecular genetics of medulloblastoma: an assessment of new therapeutic targets. Neurosurgical Review, Berlin, v. 31, n. 4, p. 359-368 2008.

CHANG, Q.; CHEN, Z.; YOU, J.; MCNUTT, M. A.; ZHANG, T.; HAN, Z.; ZHANG, X.; GONG,E.; GU,J. All-trans-retinoic acid induces cell growth arrest in a human medulloblastoma cell line. Journal of NeuroOncology, Boston, v. 84, n. 3, p. 263-267, 2007.

CHEN, Y.; STRUHL, G. Dual roles for patched in sequestering and transducing Hedgehog. Cell, Cambridge,v. 87, n. 3, p. 553-563, 1996.

CLIFFORD, S. C.; LUSHER, M. E.; LINDSEY, J. C.; LANGDON, J. A.; GILBERTSON, R. J.; STRAUGHTON, D.; ELLISON, D. W. Wnt/Wingless pathway activation and chromosome 6 loss characterize a distinct molecular sub-group of medulloblastomas associated with a favorable prognosis. Cell Cycle, Georgetown, v. 5, n. 22, p. 2666-2670, 2006.

CRAWFORD, J. R.; MACDONALD, T. J.; PACKER, R. J. Medulloblastoma in childhood: new biological advances. Lancet Neurology, London, v. 6, n. 12, p. 1073-1085, 2007.

DE HAAS, T.; OUSSOREN, E.; GRAJKOWSKA, W.; PEREK-POLNIK, M.; POPOVIC, M.; ZADRAVEC-ZALETEL, L.; PERERA, M.; CORTE, G.; WIRTHS, O.; VAN SLUIS, P.; PIETSCH, T.; TROOST, D.; BAAS, F.; VERSTEEG, R.; KOOL, M. OTX1 and OTX2 expression correlates with the clinicopathologic classification of medulloblastomas. Journal of Neuropathology and Experimental Neurology, Lawrence, v. 65, n. 2, p. 176-186, 2006.

DELLOVADE, T.; ROMER, J. T.; CURRAN, T.; RUBIN, L. L. The hedgehog pathway and neurological disorders. Annual Review of Neuroscience, Palo Alto, v. 29, p. $539-563,2006$.

DI, C.; LIAO, S.; ADAMSON, D. C.; PARRETT, T. J.; BRODERICK, D. K.; SHI, Q.; LENGAUER, C.; CUMMINS, J. M.; VELCULESCU, V. E.; FULTS, D.W.; MCLENDON, R. E.; BIGNER, D. D.; YAN, $\mathrm{H}$. Identification of OTX2 as a medulloblastoma oncogene whose product can be targeted by all-trans retinoic acid. Cancer Research, Chicago, v. 65, n. 3, p. 919-924, 2005. 
DIMARCOTULLIO, L.; FERRETTI,E.; DE SMAELE, E.; SCREPANTI, I.; GULINO, A. Suppressors of hedgehog signaling: linking aberrant development of neural progenitors and tumorigenesis. Molecular Neurobiology, Clifton, v. 34, n. 3, p. 193-204, 2006.

EBERHART, C. G.; KEPNER, J. L.; GOLDTHWAITE, P. T.; KUN, L. E.; DUFFNER, P. K.; FRIEDMAN, H. S.; STROTHER, D. R.; BURGER, P. C. Histopathologic grading of medulloblastomas: a Pediatric Oncology Group study. Cancer, New York, v. 94, n. 2, p. 552-560, 2002.

ELLISON, D. Classifying the medulloblastoma: insights from morphology and molecular genetics. Neuropathology and Applied Neurobiology, Oxford, v. 28, n. 4, p. 257-282, 2002.

FAN, X.; MIKOLAENKO, I.; ELHASSAN, I.; NI, X.; WANG, Y.; BALL, D.; BRAT, D. J.; PERRY, A.; EBERHART, C. G. Notch1 and notch2 have opposite effects on embryonal brain tumor growth. Cancer Research, Chicago, v. 64, n. 21, p. 7787-7793, 2004.

FEIDEN, S.; FEIDEN, W. WHO classification of tumours of the CNS: revised edition of 2007 with critical comments on the typing und grading of common-type diffuse gliomas. Der Pathologe, Berlin, v. 29, n. 6, p. 411$421,2008$.

FOGARTY, M. P.; KESSLER, J. D.; WECHSLERREYA, R. J. Morphing into cancer: the role of developmental signaling pathways in brain tumor formation. Journal of Neurobiology, New York, v. 64, n. 4, p. 458-475, 2005.

FRIEDMAN, H. S.; OAKES, W. J.; BIGNER, S. H.; WIKSTRAND, C. J.; BIGNER, D. D. Medulloblastoma: tumor biological and clinical perspectives. Journal of Neuro-Oncology, Boston, v. 11, n. 1, p. 1-15, 1991.

GARBER, K. Hedgehog drugs begin to show results. Journal of the National Cancer Institute, Bethesda, v. 100, n. 10, p. 692-697, 2008.

GIANGASPERO, F.; PERILONGO, G.; FONDELLI, M. P.; BRISIGOTTI, M.; CAROLLO, C.; BURNELLI, R.; BURGER, P. C.; GARRE, M. L. Medulloblastoma with extensive nodularity: a variant with favorable prognosis. Journal of Neurosurgery, Chicago, v. 91, n. 6, p. 971-977, 1999.
GIANGASPERO, F.; RIGOBELLO, L.; BADIALI, M.; LODA, M.; ANDREINI, L.; BASSO, G.; ZORZI, F.; MONTALDI, A. Large-cell medulloblastomas. A distinct variant with highly aggressive behavior. The American Journal of Surgical Pathology, New York, v. 16, n. 7, p. 687-693, 1992.

GILBERTSON, R. J.; ELLISON, D. W. The origins of medulloblastoma subtypes. Annual Review of Pathology, Palo Alto, v. 3, p. 341-365, 2008.

HALILAGIC, A.; RIBES, V.; GHYSELINCK, N. B.; ZILE, M. H.; DOLlE, P.; STUDER, M. Retinoids control anterior and dorsal properties in the developing forebrain. Developmental Biology, New York, v. 303, n. 1, p. 362-375, 2007.

HALPERIN, E. C.; FRIEDMAN, H. S. Is there a correlation between duration of presenting symptoms and stage of medulloblastoma at the time of diagnosis? Cancer, New York, v. 78, n. 4, p. 874-880, 1996.

HE, T.C.; SPARKS,A.B.; RAGO, C.; HERMEKING, H.; ZAWEL, L.; DA COSTA, L. T.; MORIN, P. J.; VOGELSTEIN, B.; KINZLER, K. W. Identification of c-MYC as a target of the APC pathway. Science, New York, v. 281, n. 5382, p. 1509-1512, 1998.

HELSETH, E.; DUE-TONNESSEN, B.; WESENBERG, F.; LOTE, K.; LUNDAR, T. Posterior fossa medulloblastoma in children and young adults ( $0-19$ years): survival and performance. Child's Nervous System, Berlin, v. 15, n. 9, p. 451455, 1999.

JENNINGS, M. T.; KAARIAINEN, I. T.; GOLD, L.; MACIUNAS, R. J.; COMMERS, P. A. TGF beta 1 and TGF beta 2 are potential growth regulators for medulloblastomas, primitive neuroectodermal tumors, and ependymomas: evidence in support of an autocrine hypothesis. Human Pathology, Philadelphia, v. 25, n. 5, p. 464-475, 1994.

JOHNSON, M. L.; RAJAMANNAN, N. Diseases of Wnt signaling. Reviews in Endocrine \& Metabolic Disorders, Boston, v. 7, n. 1-2, p. 41-49, 2006.

KATSETOS,C.D.;BURGER,P.C.Medulloblastoma. Seminars in Diagnostic Pathology, New York, v. 11, n. 2, p. 85-97, 1994. 
KATSETOS, C. D.; HERMAN, M. M.; FRANKFURTER, A.; GASS, P.; COLLINS, V. P.; WALKER, C. C.; ROSEMBERG, S.; BARNARD, R. O; RUBINSTEIN, L. J. Cerebellar desmoplastic medulloblastomas. A further immunohistochemical characterization of the reticulin-free pale islands. Archives of Pathology \& Laboratory Medicine, Chicago, v. 113, n. 9, p. 1019-1029, 1989.

KLEIHUES, P.; BURGER, P. C.; SCHEITHAUER, B. W. Histological typing of tumours of the central nervous system. Berlin: Springer-Verlag, 1993.

KLEIHUES, P.; BURGER, P. C.; SCHEITHAUER. B. W. The new WHO classification of brain tumours. Brain Pathology, Zürich, v. 3, n. 3, p. 255-268, 1993.

KOOL, M.; KORSHUNOV, A.; REMKE, M.; JONES, D. T.; SCHLANSTEIN, M.; NORTHCOTT, P. A.; CHO, Y. J.; KOSTER, J.; SCHOUTENVAN MEETEREN, A.; VAN VUURDEN, D.; CLIFFORD, S. C.; PIETSCH, T.; VON BUEREN, A. O.; RUTKOWSKI, S.; MCCABE, M.; COLLINS, V. P.; BACKLUND, M. L.; HABERLER, C.; BOURDEAUT, F.; DELATTRE, O.; DOZ, F.; ELLISON, D. W.; GILBERTSON, R. J.; POMEROY, S. L.; TAYLOR, M. D.; LICHTER, P.; PFISTER, S. M. Molecular subgroups of medulloblastoma: an international meta-analysis of transcriptome, genetic aberrations, and clinical data of WNT, SHH, Group 3, and Group 4 medulloblastomas." Acta Neuropathologica, Berlin, v. 123, n. 4, p. 473-484, 2012.

LANGOWSKI, J. L.; KASTELEIN, R. A.; OFT, M. Swords into plowshares: IL-23 repurposes tumor immune surveillance. Trends in Immunology, Oxford, v. 28, n. 5, p. 207-212, 2007.

LARSEN, K. B.; LUTTERODT, M. C.; MOLLGARD, K.; MOLLER, M. Expression of the homeobox genes OTX2 and OTX1 in the early developing human brain. The Journal of Histochemistry and Cytochemistry, Baltimore, v. 58, n. 7, p. 669-678, 2010.

LI, K. K.; LAU, K. M.; NG, H. K. Signaling pathway and molecular subgroups of medulloblastoma. International Journal of Clinical and Experimental Pathology, Madison, v. 6, n. 7, p. 1211-1222, 2013.
LOUIS, D. N.; OHGAKI, H.; WIESTLER, O. D.; CAVENEE, W. K.; BURGER, P. C.; JOUVET, A.; SCHEITHAUER, B. W.; KLEIHUES, P. The 2007 WHO classification of tumours of the central nervous system. Acta Neuropathologica, Berlin, v. 114, n. 2, p. 97-109, 2007.

MCMANAMY, C. S.; LAMONT, J. M.; TAYLOR, R. E.; COLE, M.; PEARSON, A. D.; CLIFFORD, S. C.; ELliSON, D. W. Morphophenotypic variation predicts clinical behavior in childhood non-desmoplastic medulloblastomas. Journal of Neuropathology and Experimental Neurology, Lawrence, v. 62, n. 6, p. 627-632, 2003.

MIN, H. S.; LEE, Y. J.; PARK, K.; CHO, B. K.; PARK, S. H. Medulloblastoma: histopathologic and molecular markers of anaplasia and biologic behavior. Acta Neuropathologica, Berlin, v. 112, n. 1, p. 13-20, 2006.

MORGAN, R.; HOOIVELD, M. H.; IN DER REIDEN, P.; DURSTON, A. J. A conserved 30 base pair element in the Wnt-5a promoter is sufficient both to drive its' early embryonic expression and to mediate its' repression by otx2. Mechanisms of Development, Shannon, v. 85, n. 1-2, p. 97-102, 1999.

MUOIO, V. M. F. Análise molecular dos genes OTX1 e OTX2 em meduloblastomas. 2010. 123 f. Tese (Doutorado em Ciências) - Faculdade de Medicina da Universidade de São Paulo, São Paulo. 2010.

NORTHCOTT, P. A.; KORSHUNOV, A.; WITT, H.; HIELSCHER, T.; EBERHART, C. G.; MACK, S.; BOUFFET, E.; CLIFFORD, S. C.; HAWKINS, C. E.; FRENCH, P.; RUTKA, J. T.; PFISTER, S.; TAYLOR, M. D. Medulloblastoma comprises four distinct molecular variants. Journal of Clinical Oncology, Alexandria, v. 29, n. 11, p. 1408-1414, 2011.

NOVAK, A.; DEDHAR, S. Signaling through betacatenin and Lef/Tcf. Cellular and Molecular life Sciences, Basel, v. 56, n. 5-6, p. 523-537, 1999. 
NUMASAKI, M.; WATANABE, M.; SUZUKI, T.; TAKAHASHI, H.; NAKAMURA, A.; MCALLISTER, F.; HISHINUMA, T.; GOTO, J.; LOTZE, M. T.; KOLLS, J. K.; SASAKI, H. IL-17 enhances the net angiogenic activity and in vivo growth of human non-small cell lung cancer in SCID mice through promoting CXCR-2-dependent angiogenesis. Journal of Immunology, Baltimore, v. 175, n. 9, p. 6177-6189, 2005.

PAULINO, A. C. Current multimodality management of medulloblastoma. Current Problems in Cancer, Chicago, v. 26, n. 6, p. 317-356, 2002.

PEARL, G. S.; TAKEI, Y. Cerebellar "neuroblastoma": nosology as it relates to medulloblastoma. Cancer, New York, v. 47, n. 4, p. 772-779, 1981.

PETERSON,K.;WALKER, R.W.Medulloblastoma/ primitive neuroectodermal tumor in 45 adults. Neurology, Minneapolis, v. 45, n. 3 pt 1, p. 440-442, 1995.

PIZZO, P. A.; POPLACK, D. G. Principles and practice of pediatric oncology. Philadelphia: Lippincott-Raven, 1997.

RANGARAJAN, A.; TALORA, C.; OKUYAMA, R.; NICOLAS, M.; MAMMUCARI, C.; OH, H.; ASTER, J. C.; KRISHNA, S.; METZGER, D.; CHAMBON, P.; MIELE, L.; AGUET, M.; RADTKE, F.; DOTTO, G. P. Notch signaling is a direct determinant of keratinocyte growth arrest and entry into differentiation. The EMBO Journal, Oxford, v. 20, n. 13, p. 3427-3436, 2001.

RODINI, C. O.; SUZUKI, D. E.; NAKAHATA, A. M.; PEREIRA, M. C.; JANJOPPI, L.; TOLEDO, S. R.; OKAMOTO, O. K. Aberrant signaling pathways in medulloblastomas: a stem cell connection. Arquivos de Neuro-Psiquiatria, São Paulo, v. 68, n. 6, p. 947-952, 2010.

RONCHINI, C.; CAPOBIANCO, A. J. Induction of cyclin D1 transcription and CDK2 activity by Notch(ic): implication for cell cycle disruption in transformation by Notch(ic). Molecular and Cellular Biology, Washington, v. 21, n.1 7, p. 59255934, 2001.
RORKE, L. B. The cerebellar medulloblastoma and its relationship to primitive neuroectodermal tumors. Journal of Neuropathology and Experimental Neurology, Lawrence, v. 42, n. 1, p. 1-15, 1983.

SCHIFF, D.; WEN, P. Y. Uncommon brain tumors. Neurologic Clinics, Philadelphia, v. 13, n. 4, p. 953974, 1995.

SJOLUND, J.; MANETOPOULOS, C.; STOCKHAUSEN, M. T.; AXELSON, H. The Notch pathway in cancer: differentiation gone awry. European Journal of Cancer, New York, v. 41, n. 17, p. 2620-2629, 2005.

STECCA, B.; RUIZ I ALTABA, A. The therapeutic potential of modulators of the Hedgehog-Gli signaling pathway. Journal of Biology, London, v. 1, n. 2, p. 9, 2002.

SURESH, T. N.; SANTOSH, V.; YASHA, T. C.; ANANDH, B.; MOHANTY, A.; INDIRADEVI, B.; SAMPATH, S.; SHANKAR, S. K. Medulloblastoma with extensive nodularity: a variant occurring in the very young-clinicopathological and immunohistochemical study of four cases. Child's Nervous System, Berlin, v. 20, n. 1, p. 55-60, 2004.

SZYMAS, J.; BICZYSKO, W.; GABRYEL, P.; MORKOWSKI, S. Medulloblastoma: histological evaluation and prognosis. A clinical, immunohistochemical and electron microscopic study. Child's Nervous System, Berlin, v.3, n.2, p.74-80, 1987.

TAIPALE, J.; CHEN, J. K.; COOPER, M. K.; WANG, B.; MANN, R. K.; MILENKOVIC, L.; SCOTT, M. P.; BEACHY, P. A. Effects of oncogenic mutations in Smoothened and Patched can be reversed by cyclopamine. Nature, London, v. 406, n. 6799, p. $1005-1009,2000$.

TETSU, O.; MCCORMICK, F. Beta-catenin regulates expression of cyclin D1 in colon carcinoma cells. Nature, London, v. 398, n. 6726, p. 422-426, 1999.

THIERY, J. P. Epithelial-mesenchymal transitions in tumour progression. Nature Reviews Cancer, London, v. 2, n. 6, p. 442-454, 2002. 
THOMPSON, M. C.; FULLER, C.; HOGG, T. L.; DALTON, J.; FINKELSTEIN, D.; LAU, C. C.; CHINTAGUMPALA, M.; ADESINA, A.; ASHLEY, D. M.; KELLIE, S. J.; TAYLOR, M. D.; CURRAN, T.; GAJJAR, A.; GILBERTSON, R. J.Genomics identifies medulloblastoma subgroups that are enriched for specific genetic alterations. Journal of Clinical Oncology, New York, v. 24, n. 12, p. 1924-1931, 2006.

VANDENBERG, S. R.; HERMAN, M. M.; RUBINSTEIN, L. J. Embryonal central neuroepithelial tumors: current concepts and future challenges. Cancer Metastasis Reviews, Dordrecht, v. 5 , n. 4 , p. 343-365, 1987.

WALTER, A. W.; MULHERN, R. K.; GAJJAR, A.; HEIDEMAN, R. L.; REARDON, D.; SANFORD, R. A.; XIONG, X.; KUN, L. E. Survival and neurodevelopmental outcome of young children with medulloblastoma at St Jude Children's Research Hospital. Journal of Clinical Oncology, New York, v. 17, n. 12, p. 3720-3728, 1999.

XU, P.; YU, S.; JIANG, R.; KANG, C.; WANG, G.; JIANG, H.; PU, P. Differential expression of Notch family members in astrocytomas and medulloblastomas. Pathology Oncology Research, Budapest, v. 15, n. 4, p. 703-710, 2009.

YACHNIS, A. T.; RORKE, L. B.; TROJANOWSKI, J. Q. Cerebellar dysplasias in humans: development and possible relationship to glial and primitive neuroectodermal tumors of the cerebellar vermis. Journal of Neuropathology and Experimental Neurology, Pittsburgh, v. 53, n. 1, p. 61-71, 1994.

YOKOTA, N.; MAINPRIZE, T. G.; TAYLOR, M. D.; KOHATA, T.; LORETO, M.; UEDA, S.; DURA, W.; GRAJKOWSKA, W.; KUO, J. S.; RUTKA, J. T. Identification of differentially expressed and developmentally regulated genes in medulloblastoma using suppression subtraction hybridization. Oncogene, Basingstoke, v. 23, n. 19, p. 3444-3453, 2004.

YOON, J. W.; GILBERTSON, R.; IANNACCONE, S.; IANNACCONE, P.; WALTERHOUSE, D. Defining a role for Sonic hedgehog pathway activation in desmoplastic medulloblastoma by identifying GLI1 target genes. International Journal of Cancer, New York, v. 124, n. 1, p. 109-119, 2009.
YUAN, L.; ZHANG, H.; LIU, J.; RUBIN, J. B.; CHO, Y. J.; SHU, H. K.; SCHNIEDERJAN, M.; MACDONALD, T. J. Growth factor receptorSrc-mediated suppression of GRK6 dysregulates CXCR4 signaling and promotes medulloblastoma migration. Molecular Cancer, London, v. 12, p. 18, 2013.

ZHOU, P.; SHA, H.; ZHU, J. The role of T-helper 17 (Th17) cells in patients with medulloblastoma. The Journal of International Medical Research, Northampton, v. 38, n. 2, p. 611-619, 2010.
Recebido em: 24 jun. 2014. Aceito em: 23 set. 2014. 
